\title{
People have access to implicit self-esteem unless they do not apply an ego defence
}

\author{
Aleksandra Katarzyna Fila-Jankowska \\ University of Social Sciences and Humanities, Sopot, Poland
}

BACKGROUND

Early definitions of implicit self-esteem (ISE) assumed its unconscious character. Although researchers have shown ways to achieve consistency between explicit and implicit self-esteem measures, no one has demonstrated that people may be aware of their ISE.

\section{PARTICIPANTS AND PROCEDURE}

In the experiment with 85 participants aged from 19 to 45 years a "lie detector" procedure was used to overcome the self-enhancement bias. The definition of ISE, given to participants, referred to the phenomenon, manifested in popular ISE measures.

\section{RESULTS}

In participants who were convinced that they were being assessed in the presence of a lie detector, a significant cor- relation between referred and actual ISE was shown. Individuals characterised by defensive high self-esteem in natural conditions were less accurate in ISE estimation than those with secure high self-esteem.

\section{CONCLUSIONS}

The results, demonstrating people's access to their implicit self-esteem, may have important implications for clinical, well-being, self-acceptance, or educational issues.

\section{KEY WORDS}

implicit self-esteem; awareness of implicit attitude; self-enhancement; self-deception; defensive high self-esteem

CORRESPONDING AUTHOR - Prof. Aleksandra Katarzyna Fila-Jankowska, University of Social Sciences and Humanities, 16/20 Polna Str., 81-345 Sopot, Poland, e-mail: afila-jankowska@swps.edu.pl

AUthors' CONtribution - A: Study design · B: Data collection · C: Statistical analysis · D: Data interpretation ·

E: Manuscript preparation · F: Literature search · G: Funds collection

TO CITE THIS ARTICLE - Fila-Jankowska, A. K. (2018). People have access to implicit self-esteem unless they do not apply

an ego defence. Current Issues in Personality Psychology, 6(2), 154-163.

RECEIVED 29.12.2016 · REVIEWED 02.01.2017 · ACCEPTED 21.08.2017 · PUBLISHED 18.12.2017 


\section{BACKGROUND}

Most theorists consider explicit self-esteem (ESE) and implicit self-esteem (ISE) to be two distinct but related constructs (Bosson, Swann, \& Pennebaker, 2000). ESE is assessed by means of self-reports as a conscious declaration of one's own value and is based on beliefs about the self. ISE refers to unconscious, affective associations with the self, activated automatically (Greenwald \& Banaji, 1995). ISE is obtained by indirect measurement, based on specific reactions, which are difficult to control (e.g. Gawronski, LeBel, \& Peters, 2007).

Research in recent decades has demonstrated that ISE, much like ESE, may be a significant predictor of cognitive processes and behaviours of great importance - like coping with negative feedback, unpleasant emotions, interpersonal stressors, or thoughts about death. The examination of ISE, beyond ESE alone, contributes to a deeper understanding of human nature; however, much remains to be explained in terms of the properties of this construct (Schimmack \& Diener, 2003).

Despite the conceptual assumption of its unconscious character, it is not absolutely certain that people are fully unaware of their ISE (Gawronski et al., 2007; Fazio \& Olson, 2003; Krizan, 2008). Thus, ISE could be a conscious (at least to some extent) form of self-esteem, distinct from ESE, which participants are less ready to reveal. However, so far, the awareness of ISE has not been proven, but a related phenomenon was demonstrated: under specific conditions, a coherence between ESE and ISE measures was obtained. ESE and ISE generally correlate weakly or not at all (Buhrmester, Blanton, \& Swann, 2011; Olson, Fazio, \& Hermann, 2007). In the study by Koole, Dijksterhuis, and van Knippenberg (2001), there was a stronger correlation between ISE and ESE when participants were deprived of the opportunity to reflect on their explicit responses. LeBel (2010) proved that fast ESE endorsements are more congruent with ISE. Apparently, results of automatic self-evaluative associations correlate with ESE better when the latter is estimated with limited resources.

Jordan, Whitfield, and Zeigler-Hill (2007) demonstrated ISE-ESE congruence among individuals relying on their intuition during self-assessment. Researchers argue that people who perceive intuition as a valid source of information, incorporate their ISE into their explicit self-view. They suggest that we tend to be aware of our ISE (at least to some extent) and experience it as intuition (manifested, e.g., by gut reactions). Hofmann, Gschwendner, and Schmitt (2005) have shown, in a meta-analysis, higher correlations between implicit and explicit attitudes in domains where people rely on "gut feelings."

Koole, Govorun, and Cheng (2009) proved that certain mental states can be achieved during meditation, promoting the integration of ISE and ESE. According to the neurological evidence, meditation synchronises activity in various areas of the brain and probably integrates neural processes into higher-order cognitive and affective functions. Also, the observation of one's own internal and external states without making judgments (as during meditation) may promote acceptance and awareness of both states and, consequently, the congruence between ISE and ESE. In the cited study, participants probably had access to their ISE - as evidenced by a slowdown in the process of explicit self-evaluation due to attentional resource mobilisation among the holders of low ISE. However, meditation made people stop denying it.

Therefore, one would think that people avoid being aware of low ISE or avoid "admitting" it when it is low. It has already been shown that the holders of high ESE but low ISE (or so-called defensive high self-esteem) (Jordan, Spencer, Zanna, Hoshino-Browne, \& Correll, 2003) display defensive self-enhancement, manifesting in idealised self-perception (Bosson, Brown, Zeigler-Hill, \& Swann, 2003; Jordan, Spencer, \& Zanna, 2005; Schröder-Abe, Rudolph, \& Schutz, 2007) and have weaker abilities to self-regulate emotions following failure than holders of high ESE and high ISE (or secure high self-esteem) (Kernis et al., 2008; Lambird \& Mann, 2006; McGregor \& Marigold, 2003).

\section{RESEARCH PROBLEM}

Gailliot and Schmeichel (2006) tried to verify whether people are able to realise their actual ISE. Results of their research did not confirm this ability. In the study, ISE was assessed with Nuttin's (1987) Name Letter Test (NLT) - a commonly used ISE measure (Bosson et al., 2000; Koole et al., 2001). In NLT the respondents' initial preference indicator is built on the basis of letter liking and reveals the affective associations with the self. After ISE measurement, ESE was obtained with a well-validated and reliable test - Rosenberg (1965) Self-Esteem Scale (RSES). Three months later, researchers estimated "conscious awareness of ISE" with a 12-item questionnaire, using RSES as a base, only slightly extending it and modifying questions (e.g. "On the whole, at an unconscious level, I am satisfied with myself", "On the whole, at an unconscious level, I wish I could have more respect for myself").

As a result Gailliot and Schmeichel obtained modest correlation of estimated and actual ISE: $r(257)$ $=.15, p=.050$, significant probably due to a large sample. But the estimated ISE did not correlate with the actual one when controlling for ESE. So, as the researchers conclude, without relying on ESE, participants were unable to estimate properly their ISE. However, as we postulate, it does not prove the im- 
possibility of recognizing ISE in other conditions.

Galliot and Schmeichel measured "conscious awareness of ISE" using a questionnaire, which requires extensive reflection, which could have been one of the reasons for failure to disclose the consistency between reported and actual ISE. Conscious attention to the self is driven by conceptual processing, which may inhibit more immediate feelings about the self (Silvia \& Duval, 2001; Swann, Chang-Schneider, \& McClarty, 2007). Therefore, to achieve a conscious result close to ISE, the assessment should be based on a more immediate, affective experience of the self. Such requirements would be met with a simple estimation of ESE with one scale rather than with the compound RSES-like questionnaire.

Another reason for Galliot and Schmeichel's null result could be the timing. A disadvantage of NLT is the modest temporal stability of the assessment (Buhrmester, Blanton, \& Swann, 2011), so the measure may reveal ephemeral states rather than chronic dispositions. Therefore, a better way to achieve the discussed compliance may be to ask respondents to estimate their ISE soon after assessing it with NLT.

Finally, Gailliot, and Schmeichel (2006) did not try to motivate respondents to identify their ISE, and motivation seems to be important if people are not ready to recognise or reveal their ISE. Also, the description of ISE given to participants in the cited study: "self-esteem that is beyond conscious awareness" or "how you feel about yourself deep, deep, inside of you" could have been unclear. Thus, the lack of connection between actual and reported ISE does not prove the lack of possibility to recognise ISE properly. Therefore, our first research question is: can we identify the conditions under which people report their ISE consistently with its actual value? If we could find an example of such conditions, logically it would be correct to say that there are circumstances in which people are able to recognise their ISE.

Our second question is whether individuals with secure high self-esteem estimate their ISE more accurately than people with defensive high self-esteem. Gailliot and Schmeichel (2006) assumed the latter because people who are threatened by aversive circumstances apply an ego defence and deny their low ISE. This may manifest as overestimation of their ISE level - in opposition to the holders of secure high self-esteem, who would not avoid acknowledging their actual level of ISE and hence estimate it more accurately than their counterparts. The results of the cited study did not confirm this assumption as well - which is understandable if the appropriate conditions for the disclosure of ISE were not present. So, in the presented study we addressed the same question.

We decided to repeat the Gailliot and Schmeichel (2006) study with some modifications including the above claims. Finding the right method to reinforce respondents' motivation to recognise (or reveal) their
ISE was preceded by pilot studies, as well as finding an ISE definition understandable to respondents.

\section{RESEARCH DESIGN}

Bosson et al. (2000) compared the ISE construct with an elephant, which may seem like something else, depending on which side of it is being touched. Although researchers differ in how they understand an affective association within self-representation as a base for ISE, they agree that implicit measures similar to NLT tap into the first self-evaluation that comes to mind (Buhrmester et al., 2011). We used this fact when preparing an ISE definition.

Two pilot studies (attended by a total of 124 students of different fields at several universities, aged 18 to 60 years) were conducted to find an appropriate definition and reinforcement. As for definitions in Study 1, we referred to a concept by Burhmester, Blanton, \& Swann (2011), who defined ISE as a latent, or hidden self-evaluation, which people are unable or unwilling to report. In Study 2, we directed participants' attention to their "gut feelings" as a source of ISE - in reference to Jordan, Whitfield, \& Zeigler-Hill (2007) research, we defined it as "a gut feeling, prompted by the body".

Different reinforcements to guess ISE were also examined - in Study 1, three experimental conditions were applied: In the first one participants were informed "research results show that people who recognise their implicit self-esteem experience greater satisfaction in their lives; implicit self-knowledge is especially valuable in partner relationships" (compare Jordan, Whitfield, \& Ziegler, 2007, Study 4). In the second one, the words "greater satisfaction" were changed to "less satisfaction". In the control condition participants did not get any information about the value of ISE knowledge. Because these three conditions did not differentiate participants in terms of their accuracy in recognising ISE, in Study 2 only the incentive "Are you able to guess your implicit self-esteem?" was applied, compared to the lack of it. This incentive was the most promising. It presents ISE knowledge as a kind of competence, without emphasising its value or its drawbacks, which can cause too much effort to achieve or avoid the proper ISE.

Despite getting close to significance level, none of the samples showed a significant correlation between the reported and actual ISE. It may be related to a suspected, impaired awareness of ISE due to self-deception or an unreadiness to declare it due to self-presentation. To reduce both these reactions, we decided to convince the respondents that their ISE is available not only to them but also to the experimenter. We designed the experiment in the bogus pipeline procedure (Kassin, Fein, \& Markus, 2008). 


\section{HYPOTHESES}

H1. People report their ISE consistently with its actual value under the conditions of being motivated to do so and having an understanding of the ISE phenomenon. H2. People with secure high SE are more accurate in estimating their ISE than those with defensive high SE.

\section{PARTICIPANTS AND PROCEDURE}

Eighty-five people (39 men and 46 women) of different professions (with university or college education) aged from 19 to 45 years $(M=28.31, S D=7.63)$ took part in the study, which was held in a separate room during a large social gathering.

\section{MEASURES AND PROCEDURE}

At the outset, ISE was measured using the NLT procedure, which requires the participants to rate how much they like each letter of the alphabet on a scale from 1 (I dislike it very much) to 7 (I like it very much). ISE scores were computed in a commonly used procedure (Kitayama \& Karasawa, 1997): 1. Calculate an average preference for each letter based on the ratings of individuals who do not have these letters in their initials; 2 . Subtract this "normative score" from participants' rating of each initial; and 3. Average the values obtained for two initials. The higher the score, the higher the person's ISE.

Then we measured participants' ESE with a single 10 -point scale (from very low to very high). In the next step the respondents who were assigned (randomly) to the experimental group were connected to a pulse oximeter through its handle via a sensor on their finger, and were asked to read the following instruction: In a moment, you will be asked to assess yourself while being connected to a device that measures physiological indicators of arousal (to verify your answers). The test is painless. In the control condition,

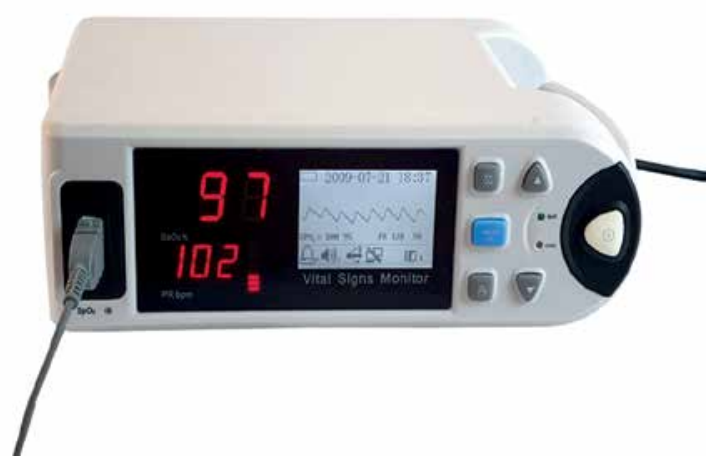

the instruction was short: In a moment you will be asked to assess yourself. Then, participants filled the RSES questionnaire and read the identical information: Implicit self-esteem is the first feeling about the self, positive or negative, that you experience upon suddenly hearing your name. Are you able to correctly identify this implicit feeling? Try to estimate it on the following 10-point scale. Before debriefing, participants from the experimental group were asked what they thought the device was. The most common answer was "A lie detector". Nobody recognised the manipulation.

The pulse oximeter used (portable, compact, OXY 9, made by BIONET company, with LCD display, battery powered - Figure 1) noninvasively measures blood oxygen saturation. During the measurement, the device displays changing numbers and lines, which convince the respondents that they are being subjected to a real measurement.

The measurement of ESE before manipulation, made to identify secure and defensive self-esteem, was as short as possible to avoid excessive self-reflection that may further affect measurements. It is well documented that SE estimation on a single scale correlates highly with the RSES index (Robins, Hendin, \& Trzesniewski, 2001). On the other hand, ESE was traditionally measured after manipulation to see if there was an incorporation of ISE into ESE (manifested as the correlation of these two levels of self-evaluation) as a result of "lie detection".

ISE scores were computed in a commonly used procedure (Kitayama \& Karasawa, 1997) after respondents assessed the attractiveness of each letter of the alphabet (in NLT procedure).

\section{RESULTS}

The reliability of the RSES was high: Cronbach $\alpha=$ .81. There was no difference between the groups in two kinds of self-esteem measured before manipulation - ESE on a 10-point scale and ISE by NLE.

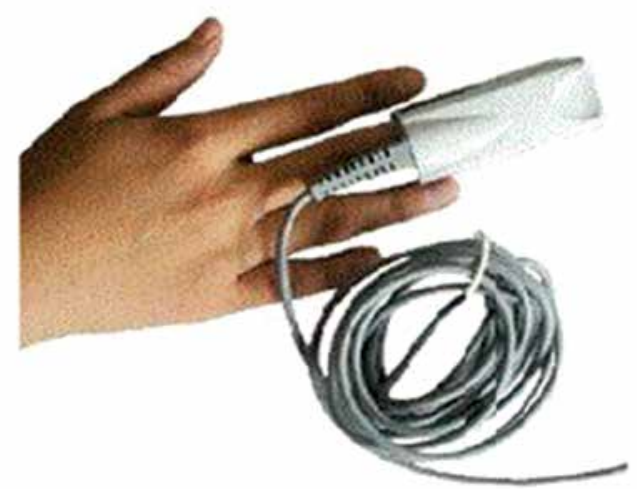

Figure 1. The pulse oximeter used in the study. 
Table 1

Correlations between reported ISE and measures of self-esteem. Values in parentheses are partial correlations while controlling for ESE measured before manipulation and for ESE measured after manipulation

\begin{tabular}{|c|c|c|c|c|c|}
\hline Measure & $M$ & $S D$ & $\begin{array}{c}\text { ESE } \\
(10-\text { point scale })\end{array}$ & $\begin{array}{c}\text { ESE } \\
(\mathrm{RSES})\end{array}$ & $\begin{array}{c}\text { ISE } \\
(\mathrm{NLE})\end{array}$ \\
\hline \multicolumn{6}{|l|}{ Pulse oximeter present } \\
\hline reported ISE & 5.99 & 1.11 & $.65^{* *}$ & $.76^{* *}$ & $.38^{*}\left(.35^{*}\right)(.27 \#)$ \\
\hline ESE (10-point scale) & 6.38 & 1.21 & 1 & $.74^{* *}$ & .19 \\
\hline ESE (RSES) & 4.21 & .63 & 1 & $.28^{*}$ & \\
\hline ISE (NLE) & 1.24 & 1.14 & 1 & & \\
\hline \multicolumn{6}{|l|}{ Pulse oximeter absent } \\
\hline reported ISE & 6.63 & 1.48 & $.60^{* *}$ & $.27 \#$ & $.01(-.12)(.00)$ \\
\hline ESE (10-point scale) & 6.61 & 1.37 & 1 & $.59^{* *}$ & .17 \\
\hline ESE (RSES) & 4.06 & .62 & 1 & .06 & \\
\hline ISE (NLE) & 1.01 & .96 & 1 & & \\
\hline
\end{tabular}

Note. ${ }^{* *} p<.01 .{ }^{*} p<.05$; two-tailed test; $\# p<.05$, one-tailed test.

The use of the pulse oximeter did not affect the mean level of ESE measured with RSES (average scores are shown in Table 1).

When the reported ISE was submitted to the 2 (oximeter: absent, present) $\times 2$ (gender) ANCOVA with the age as covariate, the main effect of the oximeter condition was revealed: $F(1,78)=4.77, p=.050$, which informed that people connected to the device reported a lower level of ISE than those who were not connected. Gender and age control were applied due to the often-reported association of these variables with self-esteem. Covariate was entered in order to statistically equalise the subjects in terms of age.

Correlations between the measured variables inside the groups of manipulation are shown in Table 1.

According to Hypothesis 1, in the group tested with the pulse oximeter, the reported ISE and the actual ISE were positively and significantly correlated. They remained significant while controlling for ESE measured both before and after manipulation. In the control condition, none of these correlations were significant.

Pre-manipulation measures of ISE and ESE allowed for verification of Hypothesis 2. We computed a measure of accuracy in estimating ISE (compare Gailliot \& Schmeichel, 2006) as the absolute value of the difference between standardised actual ISE score and standardised reported ISE score. Lower magnitude of this discrepancy indicated greater accuracy in ISE evaluation ${ }^{1}$. The accuracy score was regressed on the standardised ISE, standardised ESE, condition of the oximeter $(+1$ - present, $-1-$ absent $)$, and four interaction terms, generated by creating cross-product vectors: ISE $\times$ ESE, ESE $\times$ oximeter condition, ISE $\times$ oximeter condition, ISE $\times$ ESE $\times$ oximeter condition (Aiken \& West, 1991). The model was significant: $F(7,75)=4.22, p<.001$. The regression summary is presented in Table 2 .

Table 2

Regression summary of accuracy in estimating ISE

\begin{tabular}{lccccc}
\hline & $B$ & $\begin{array}{c}\text { Standard } \\
\text { error }\end{array}$ & $\beta$ & $t$ & $p$ \\
\hline ISE & -.06 & .08 & -.08 & -.78 & .44 \\
ESE & -.15 & .08 & -.19 & -1.77 & .08 \\
Oximeter condition & -.10 & .08 & -.13 & -1.33 & .19 \\
ISE $\times$ ESE & .32 & .07 & -.47 & -4.32 & .00 \\
ISE $\times$ oximeter condition & .05 & .08 & .07 & .64 & .52 \\
ESE $\times$ oximeter condition & -.03 & .08 & -.04 & -.33 & .74 \\
SE $\times$ ESE $\times$ oximeter condition & .15 & .07 & .22 & 1.97 & .05 \\
\hline
\end{tabular}



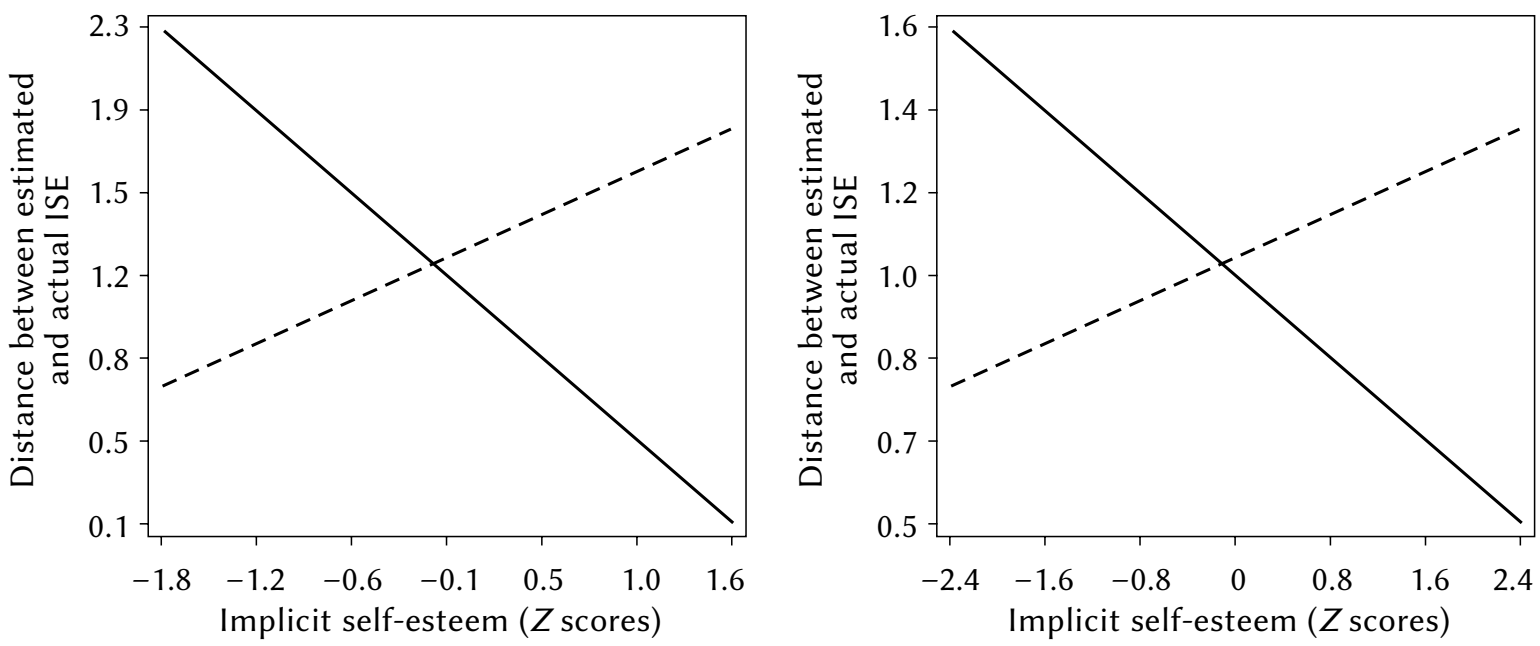

Explicit SE - high - - low

Figure 2. The absolute difference between actual and estimated ISE as a function of the level of ISE and ESE in the group tested without the pulse oximeter (the left panel) and with the pulse oximeter (the right panel).

The significant second-order interaction effect of ISE and ESE was qualified with the marginally significant third-order interaction of ISE, ESE, and oximeter condition.

In the control condition, the interaction of ESE and ISE was significant in explaining the accuracy of ISE estimation: $\beta=-.46, t(3,42)=-3.73, p<.001$ (Figure 2 - left panel).

Simple-slope analyses revealed that among people with high (+1 SD) ESE, the level of ISE was significantly related to the accuracy indicator: $B=-.64$, $t(42)=-4.06, p<.001$, but among those with low $(-1 \mathrm{SD})$ ESE, this relation was not significant, with a tendency for the opposite: $\mathrm{B}=.33, p=.060$. Thus, participants with a secure high SE reported ISE more accurately than participants with defensive high SE, which confirms Hypothesis 2.

In the oximeter condition, the interaction of ESE and ISE was also significant in explaining the accuracy of ISE estimation: $\beta=-.17, t(3,33)=-2.16, p=.030$ (Figure 2 - right panel), but both simple slopes were insignificant: $B=-.15, p=.240$ for high ESE, and $B=.16, p=.110$ for low ESE. Therefore, Hypothesis 2 is no longer true after the bogus pipeline procedure.

\section{DISCUSSION}

In the presented study, we attempted to check whether people are able to properly evaluate their implicit self-esteem. Despite the conclusion of Gailliot and Schmeichel (2006), inferred on the basis of their research, we assumed that it is possible, albeit with proper motivation and an accurate explanation of what implicit self-esteem is, to measure implicit self-esteem. The study was conducted in the scheme utilised by Gailliot and Schmeichel (2006) - first ISE was measured, then ESE was measured, and finally respondents were asked to assess their implicit self-esteem. Also, we used the same tools as the aforementioned researchers to measure ISE and ESE - i.e. Nuttin's Name Letter Test and Rosenberg's Self-Esteem Scale. However, significant modifications were introduced. The main dependent variable, i.e. ISE reported by the respondents, was measured on one scale, rather than with a questionnaire that requires reflection. According to Swann, Chang-Schneider, and McClarty (2007), automatic self-evaluations (or implicit SE) based on immediate feelings about the self become inhibited in the process of rational thinking, which uses linguistic categories and cognitive schemas. Hence, we assumed that actual ISE could correlate with rapidly obtained, therefore highly accessible, explicit SE and, more importantly for the design of the research, with a simple ISE estimation (which is a kind of explicit self-assessment as well). Another change in the Gailliot and Schmeichel study construction was the ISE estimation (by participants) only a short time after the initial measurement of self-esteem because, according to contemporary knowledge, any measure of ISE is always a measure of a transient state of self-esteem rather than a constant trait (Buhrmester et al., 2011).

The overall picture of the results leads to the following view: after being given a definition of ISE, people focus on the phenomenon described there and experience some kind of feelings (even on the level of an unconscious affect). This focus on feelings can lead to an incorporation of them into ESE when the focus is sufficiently strong (for example, when it was validated as important). It cannot be excluded that such incorporation takes place without conscious control of the subject. Thus, it appears that subjects distort declarations of the (at least in part) conscious 
ISE, when for some reason they do not want to disclose it. The consistency of all three self-evaluations in the group examined in the Bogus Pipeline condition may indicate that the bias in ISE estimation is controlled, to some extent, because participants, forced to be closer to the truth, reduce this bias. Thus, the thesis of Jordan et al. (2003, 2005), that implicit $\mathrm{SE}$ is preconscious, occasionally entering awareness, seems reasonable in the face of the obtained results.

The model of APE (associative-propositional evaluation) by Gawronski and Bodenhausen $(2006,2007)$ is also consistent with this way of thinking. The central assumption in this model is that implicit attitudes reflect the affective associations in memory, whereas explicit attitudes reflect the outcome of validation processes (also compare Strack \& Deutsch, 2004). The associations are activated independently of whether a person considers them as accurate or inaccurate, but the validation process, which involves reasoning, verifies the usefulness of an activated association on the basis of subjective assessment. If the result of this assessment is consistent with other information taken into regard, it is used for the construction of the explicit attitude. If not, associatively activated evaluations are rejected as a valid basis for an explicit attitude. In such a case, implicit attitude (here ISE) can only be revealed in indirect measures. The presented results fit this model, suggesting, in addition, that people can have conscious access to the result of an associative process because an incorporation of associative evaluation can be induced in the validation process.

In the "enhanced use" model, proposed by Koole, Govorun, and Cheng (2009), there is also an assumption that people have access to their ISE. The authors argue that meditation in their study increased the extent to which respondents used an accessible feeling of ISE in the formation of ESE.

As noticed by Buhrmester et al. (2011), the assumption of ISE awareness may help to explain why individuals with high explicit but low implicit SE are defensive. Koole et al. (2009) has suggested that when those people become aware of their relatively low ISE, they experience an aversive inconsistency within the self. Thus, they are motivated to deny their negative ISE and affirm their positive ESE, and this is what triggers their defensive reactions. Koole et al. also suggest that these reactions may be adaptive in some ways - if such individuals are aware of their low ISE, but perceive it as invalid, then the latter conviction serves to protect them from depression.

Following Gailliot and Schmeichel (2006), we assumed as well that people with a secure high SE are more accurate in estimating their ISE than those with a defensive high SE. Using the accuracy indicator proposed by these researchers (this is the mathematical distance between evaluated and actual ISE), the discussed assumption was confirmed in the pre- sented study, in contrast to the research of Gailliot and Schmeichel (2006). People with consistent, high explicit, and high implicit SE were more accurate in assessing their ISE than those with high explicit, but low implicit SE. This effect was obtained in the form of the significant second-order interaction effect of ISE and ESE for the whole study group (the pattern of this interaction was almost identical to that in the left panel of Figure 2). However, if we made a separate analysis in the two experimental conditions, in the control group we gained significant confirmation of Hypothesis 2, but in the Bogus Pipeline group, people with defensive high SE were no longer less accurate in ISE estimation than those with secure high SE. Most likely the former stopped "trying to escape" from the assessment of their actual ISE, giving up self-presentation - to the extent to which the ISE was conscious. Therefore, the conclusion that ISE (at least occasionally) enters awareness also seems true in light of the results concerning the correctness of ISE guessing. Regardless, defensiveness in people with high ESE and low ISE in the presented study also seems to be confirmed.

\section{LIMITATIONS}

A serious limitation of the presented study is the fact that only one measure of implicit self-esteem was used - namely Nuttin's Name Leter Test. NLT was originally conceptualised as a measure of implicit egotism rather than self-esteem (Buhrmester et al., 2011) Pelham, Mirenberg, and Jones (2002), and some researchers make a distinction between the tendency to display positivity biases (or implicit egotism) and self-esteem (e.g. Kwang \& Swann, 2010). Currently, measurement of this commonly used measure of ISE is based on the assumption that an affect connected to the self, spills over into evaluations of objects associated with the self. The NLT turned out to be one of two (along with SE-IAT) of the most valid and reliable measures of ISE (Bosson et al., 2000; Koole et al., 2001) and as such was also used in the study of Gailliot and Schmeichel (2006). Because we refer to this study, we also use the same measure, of which the limitations are known. As Buhrmester et al. (2011) highlight, the NLT focuses on respondents' feelings associated with a specific aspect of self-regard, i.e. their initials, and we can only hope that people's affect towards their initials reflects their global feelings of self-worth. This may be true to some degree, along with the assumption that the NLT taps into unconscious processes, because - as previously shown nearly half of the respondents were able to figure out what the purpose of NLT was. However, one has to start with something, and it is comforting that it was possible to show the correctness of ISE recognition, measured even with the NLT. This correctness might 
be so low (outside causes inherent in the procedure) because of the fact that ISE shows a particular aspect of self-esteem. On the other hand - the obtained correlation of evaluated (by participants) ISE and ISE as measured with NLT provides some support for the assumption that the NLT measures (at least partially) what it is supposed to measure - this is an effect of the first associations with the self (because the ISE was described in this way in the definition given to participants). The obtained results obviously require replication using a different measure of implicit self-esteem.

Perhaps a better solution in study would be to connect both groups to the pulse oximeter, and to explain to only one of them that physiological indicators of arousal are measured in order to verify the honesty of their answers. On the other hand, no explanation of "plugging" in the control group, or an explanation that the measurement is independent of subjects' answers, might arouse suspicion and consequently even more anxiety than in the experimental group.

Another limitation of the study was narrowing down the comparisons of accuracy of ISE estimation to only two groups, designated by the consistency vs. inconsistency of ISE and ESE. However, we did not find sufficient grounds in the literature to assume how correct the estimation of ISE in the other two groups would be - i.e. in the group with low ESE and low ISE and in the group with low ESE but high ISE. The analyses performed in our study have shown that the accuracy of ISE estimation was in the range designated by the correctness of the group with defensive high SE and the group with secure high SE, but the differences from non-analysed groups did not reach the level of significance.

\section{FUTURE DIRECTIONS}

Although the aim of the presented study was to show only examples of conditions for a significant connection between declared and actual ISE, perhaps one can narrow the circumstances in which this relationship arises. For example, maybe it can occur among people subjected to the Bogus Pipeline condition, who are not encouraged to explore their ISE, but the declaration of implicit self-esteem is simply required from them (notwithstanding the definition). When considering the obtained result, it is important to recognise the fact that for the first time such circumstances were shown. The purpose of a subsequent replication could be a reduction in these circumstances until the necessary condition for a correct declaration of ISE is found. An appropriate modification of these circumstances is likely to lead also to a closer connection between the actual and estimated ISE because we know that this connection can be significant.
In the discussion, we suggest that an affect, or feeling such as a "gut feeling", mediates the relationship between ISE and ESE and the relationship between ISE and its estimation.

This idea of mediation also seems to be consistent with the model of APE by Gawronski and Bodenhausen (2006) as well as with the "enhanced use" model by Koole et al. (2009).

However, the mediator of the discussed relations is probably not a declared (i.e. burdened by self-presentation) feeling of anxiety. Perhaps, according to the previous studies (e.g. Spalding \& Hardin, 1999; Conner $\&$ Barrett, 2005), it can be expected that the measure of implicit anxiety or spontaneous affect would correlate with ISE, not anxiety as reported by respondents. Thus, in the replication of the study, researchers might look for a mediator of an affective characteristic, measurable as "implicit anxiety" or "spontaneous affect".

The results of the study suggest that the moderator of the connection between ISE and ESE is the value attributed to ISE recognition. Only at high values did the incorporation of ISE into ESE take place. But such high values did not encourage participants to correctly declare their ISE. The moderator of that correct declaration seems to be the value of a sincere (true) statement of ISE (participants may consider it worthwhile to admit to their ISE if the distortion can be visible). An important element of further research may be testing these (and other) moderators of the discussed relations in the context of phenomena such as self-deception and self-presentation. Moreover, the procedure of the current study does not allow to exclude the possibility that the consistency of SE measures results from the focusing attention on physiology (which, in turn, forces greater insight). Further studies are needed to clarify the mechanism of acquisition and abandonment of self-deception or self-presentation in the process of self-evaluation. The results may have important implications for clinical issues.

\section{ENDNOTES}

1 A second accuracy rate, used by Gailliot and Schmeichel, was not created because it is correlated with the actual ISE by definition.

\section{RefERENCES}

Aiken, L. S., \& West, S. G. (1991). Multiple regression: Testing and interpreting interactions. London: Sage Publications.

Bosson, J. K., Brown, R. P., Zeigler-Hill, V., \& Swann, W. B. (2003). Self-enhancement tendencies among people with high explicit self-esteem: The moderating role of implicit self-esteem. Self and Identity, 2, 169-187. 
Bosson, J. K., Swann, W. B., Jr., \& Pennebaker, J. W. (2000). Stalking the perfect measure of implicit self-esteem: The blind men and the elephant revisited? Journal of Personality and Social Psychology, 79, 631-643.

Buhrmester, M.D., Blanton, H, \& Swann, W. B. (2011). Implicit self-esteem: Nature, measurememt, and a new way forward. Journal of Personality and Social Psychology, 100, 365-385.

Conner, T., \& Barrett, L. F. (2005). Implicit self-attitudes predict spontaneous affect in daily life. Emotion, 5, 476-488.

Fazio, R. H., \& Olson, M. A. (2003). Implicit measures in social cognition research: Their meaning and use. Annual Review of Psychology, 54, 297-327.

Gailliot, M. T., \& Schmeichel, B. J. (2006). Is implicit self-esteem really unconscious? Implicit self-esteem eludes conscious reflection. Journals of Articles in Support of the Null Hypothesis, 3, 73-83.

Gawronski, B., \& Bodenhausen, G. V. (2006). Associative and propositional processes in evaluation: An integrative review of implicit and explicit attitude change. Psychological Bulletin, 132, 692-731.

Gawronski, B., LeBel, E. P., \& Peters, K. R. (2007). What do implicit measures tell us? Validity of three common assumptions. Association for Psychological Science, 2, 181-193.

Greenwald, A. G., \& Banaji, M. R. (1995). Implicit social cognition: Attitudes, self-esteem, and stereotypes. Psychological Review, 102, 4-27.

Hofmann, W., Gschwendner, T., \& Schmitt, M. (2005). On implicit - explicit consistency: The moderating role of individual differences in awareness and adjustment. European Journal of Personality, 19, 25-49.

Jordan, C. H., Spencer, S. J., Zanna, M. P., Hoshino-Browne, E., \& Correll, J. (2003). Secure and defensive high self-esteem. Journal of Personality and Social Psychology, 85, 969-978.

Jordan, C. H., Spencer, S. J., \& Zanna, M. P. (2005). Types of high self-esteem and prejudice: How implicit self-esteem relates to ethnic discrimination among high explicit self-esteem individuals. Personality and Social Psychology Bulletin, 31, 695-700.

Jordan, C. H., Whitfield, M., \& Zeigler-Hill, V. (2007). Intuition and the correspondence between implicit and explicit self-esteem. Journal of Personality and Social Psychology, 93, 1067-1079.

Kassin, S. M., Fein, S., \& Markus, H. R. (2010). Social Psychology ( $8^{\text {th }}$ ed.). Boston, MA: Houghton Mifflin.

Kernis, M. H., Lakey, C. E., \& Heppner, W. L. (2008). Secure versus fragile high self-esteem as a predictor of verbal defensiveness: Converging findings across three different markers. Journal of Personality, 76, 477-512.

Kitayama, S., \& Karasawa, M. (1997). Implicit self-esteem in Japan: Name letters and birthday num- bers. Personality and Social Psychology Bulletin, 23, 736-742.

Koole, S. L., Dijksterhuis, A. \& van Knippenberg, A. (2001). What's in a name: Implicit self-esteem and the automatic self. Journal of Personality and Social Psychology, 80, 669-685.

Koole, S. L., Govorun, O., Cheng, C. M., \& Gallucci, M. (2009). Pulling your self together: Meditation promotes congruence between implicit and explicit self-esteem. Journal of Experimental Social Psychology, 45, 1220-1226.

Krizan, Z. (2008). What is implicit about implicit self-esteem? Journal of Research in Personality, 42, 1635-1640.

Kwang, T., \& Swann, W. B., Jr. (2010). Do people embrace praise evenwhen they feel unworthy? A review of critical tests of self-enhancement versus self-verification. Personality and Social Psychology Review, 14, 263-280.

Lambird, K. H., \& Mann, T. (2006). When do ego threats lead to self-regulation failure? Negative consequences of defensive high self-esteem. Personality and Social Psychology Bulletin, 32, 11771187.

LeBel, E. P. (2010). Attitude accessibility as a moderator of implicit and explicit self-esteem correspondence. Self \& Identity, 9, 195-208.

McGregor, I., \& Marigold, D. C. (2003). Defensive zeal and the uncertain self: What makes you so sure? Journal of Personality and Social Psychology, 85, 838-852.

Nuttin, J. M. (1987). Affective consequences of mere ownership: The name letter effect in twelve European languages. European Journal of Social Psychology, 17, 381-402.

Olson, M. A., Fazio, R. H., \& Hermann, A. D. (2007). Reporting tendencies underlie discrepancies between implicit and explicit measures of self-esteem. Psychological Science, 18, 287-291.

Pelham, B. W., Mirenberg, M. C., \& Jones, J. T. (2002). Why Susie sells seashells by the seashore: Implicit egotism and major life decisions. Journal of Personality and Social Psychology, 82, 469-487.

Robins, R. W., Hendin, H. M., \& Trzesniewski, K. H. (2001). Measuring global self-esteem: Construct validation of a single-item measure and the Rosenberg Self-Esteem Scale. Personality and Social Psychological Bulletin, 27, 151-161.

Rosenberg, M. (1965). Society and the adolescent self-image. Princeton, NJ: Princeton University Press.

Schimmack, U., \& Diener, E. (2003). Predictive validity of explicit and implicit self-esteem for subjective well-being. Journal of Research in Personality, 37, 100-106.

Schröder-Abe, M., Rudolph, A., \& Schutz, A. (2007). High implicit self-esteem is not necessarily advantageous: Discrepancies between explicit and implicit self-esteem and their relationship with anger 
expression and psychological health. European Journal of Personality, 21, 319-339.

Silvia, P. J., \& Duval, T. S. (2001). Objective self-awareness theory: Recent progress and enduring problems. Personality and Social Psychology Review, 5, 230-241.

Spalding, L. R., \& Hardin, C. D. (1999). Unconscious unease and self-handicapping: Behavioral consequences of individual differences in implicit and explicit self-esteem. Psychological Science, 10, 535-539.

Strack, F., \& Deutsch, R. (2004). Reflective and impulsive determinants of social behavior. Personality and Social Psychology Review, 8, 220-247.

Swann, W. B., Jr., Chang-Schneider, C., \& McClarty, K. L. (2007). Do people's self-views matter? Self-concept and self-esteem in everyday life. American Psychologist, 62, 84-94. 